\title{
Aesthetic Level to Explore the Relationship between College Students and the Overall Quality
}

\author{
Wang Juncheng \\ Energy and Power Engineering, Northeast Electric Power University, Jilin, Jilin, China
}

\begin{abstract}
Among the students' comprehensive quality, aesthetic quality is a very important component, it is not only the quality of the commitment of all qualities should play an independent role, but also for the improvement of the quality of other aspects also play a vital role. The aesthetic level as a measure of an important indicator of the level of aesthetic quality, to a large extent, it can also measure the overall quality level of college students.
\end{abstract}

Keywords - College students, comprehensive quality, aesthetic qualities, aesthetic level

\section{大学生审美水平与综合素质关系探究}

\author{
王俊成 \\ 东北电力大学能源与动力工程学院, 吉林, 吉林, 中国
}

摘 要 在大学生综合素质当中, 审美素质是一项十分重要的组成部分, 不仅承担了所有素质中独立的素质应当起到的作用, 而 且对于其它方面的素质提高也起着至关重要的作用。而审美水平做为衡量审美素质高低的重要指标, 很大程度上来说, 它亦能衡量出 大学生的综合素质水平。

关键词 大学生，综合素质，审美素质，审美水平

\section{1. 引言}

作为大学生综合素质的重要组成部分, 审美素质能够 起到自身应该有的作用同时, 亦能够对其它方面的素质起 到促进提高的作用。具体来说, 审美素质在我国当今物质 文明发展到一个很高的水平背景之下, 它事关新世纪大学 生自我形象完善, 以及全面发展水平。简要的说审美素质, 它与大学生是否能够发现什么是美, 美在何处, 具有多少 美都息息相关。而取决于这种发现“是什么”、“在哪里”、“有 多少”的水平就是审美水平。综合素质当中审美素质是一项 不可获取的素质, 因为基于它才能够使得大学生不断完善 发展其他方面的素质, 这样看来审美水平与综合素质的关 系就清晰起来。

\section{2. 审美的解读}

通俗的讲, 爱美之心人皆有之, 美是社会中每个人所 共同追求的目标。美的东西无时不有, 无处不在, 这便在 客观上促使人们在情感上产生对美的触感, 产生了体验美、 感受美的实践活动。当一个人被某种事物所独有的特质所
吸引, 便会主观能动的去进行对这种事物的品味和鉴赏, 并在美学、情感层面上做出判断, 这个过程活动便可成为 审美活动。审美活动直接所导致的结果就是能够使得审美 活动的主体产生对事物的美感, 既主体对客体产生的一种 认同感、契合感及愉悦感。从生理角度来看, 审美活动就 是审美主体通过对外部事物的感官上触觉, 然后经由听觉、 视觉等器官将接受的信息发送至大脑, 经过大脑的分析判 断一旦与主体自身曾经经历的美好信息发生共鸣, 那么就 会重新将主体大脑内存储的信息调动起来与之发生情感上 的共鸣。审美做为人们知晓却在日常生活中很难感受到的 一种行为, 它从古至今都一直伴随人们的客观实践活动过 程中, 它是人们与自然之间和谐共处的纽带。

在人类的审美活动过程中, 人类一方面按照美的规律 合理优化客观世界, 使得世界向着更加具有美感向着, 更 加美好的方向发展; 但同时美的世界也促使人类具有更好 的生存环境, 更好的素质, 总之审美活动使得人类与客观 世界会更加和谐的想出。从某种意义上看, 人类不断前行 
的文明正是这种具有辩证特质的审美活动使然。

在审美活动中, 主体的审美基本水平, 也就是审美素 质指的就是在该活动中所需基本的基本先天和后天素质, 主要是由审美感官、审美态度、审美趣味、审美经验等组 成

所谓审美感官指的就是参与审美活动中的个体的一些 感知美、审视美的生理特征, 如对音乐欣赏的耳朵、欣赏 绘画的眼睛, 这些都是个体审美的先天性审美素质, 而与 之相对应的是后天的审美态度、审美趣味、审美经验等所 构筑出的情趣、能力、经验等基本素养。

审美态度则是人们在从事审美活动前的一种心态表 征, 这种表征与之现实生活中的心态不同, 它可说是一种 高于世生活的超脱心态。在审美活动中, 人们要想感受客 体的美感首先就要对审美对象采取非功利的态度, 只有这 样才能细嚼慢咽似的发觉其深层次的美感, 去细细品味与 人相联系的意味、情调及精神境界等。

审美经验指的则是存在于先于付诸对审美对象进行审 美活动主体大脑之中, 能够联系对象的形象以及相关特征, 调动大脑中的记忆, 对审美对象进行感知。审美经验属于 后天性的审美素质, 它通常在审美实践活动积累出的感官 印象。这些存储出来的映像当再次进行审美活动时, 碰到 相关对象的时候会自动的调动起来。

审美情趣指的则是在审美实践活动中形成对审美对象 的主观态度、趣味与追求, 既是主体对客观审美对象的感 性的上的取向。它所反映出的是审美主体的审美需要和审 美对象之间的关系。与之审美经验类似, 审美情趣亦是在 后天审美活动中逐渐培养出来的, 但有区别的是他具有明 显的主观特性, 就是审美情趣有正面和负面之分, 好的审 美情趣能够促使人们向着更加积极的方向发展, 而负面的 审美情趣则是会使得人们的价值观发生不良的扭曲。

而审美能力则可以说是对上述有关审美相关概念的一 种综合性指标, 他与审美水平可以说的差不多是一个意思, 他对人们认识美、鉴赏美的综合水平, 具体的包括美的感 受力、理解力以及想象力等。

\section{3. 大学生综合素质与审美水平关系探讨}

大学生做为国家未来发展的中流砥柱, 这个群体的素 质高低极大程度上决定国家未来发展走向。培养大学生综 合素质的课题眼下已经成为热门话题, 特别是随着国民经 济日益发展, 外国拜金主义意识流流入冲击之下, 培养提 高大学生的综合素质已然成为必须要放在日程上的议题。 而审美素质作为大学生综合素质中重要的一项素质, 它的 水平高低可以作为一杆标尺, 来衡量大学生的综合素质。

\section{1 大学生审美素质水平的高低与其创新能力有关}

大学生审美水平高低可以对其创新能力产生重大影 响。当时间轴线跨入二十一世纪这个门槛, 时代特征已经 悄然发生了变化, 既随之当今全球化进程的日益加快, 已 然进入到了一个经济时代。在知识经济时代里什么最重要 毫无疑问是创新。在这个时代中, 知识资源生产、使 用、消费已然成为时代经济发展的原动力。做为一种长久 性资源, 知识的更新同样已经成为推动时代发展的巨大动 力, 而知识的产生需要的就是创兴。而审美素质的水平, 不仅是局限于诗词歌赋的品味当中, 在各个领域内这个内 涵都有着不同的外延。如在科技领域, 将来的大学生人才 具有高水平的审美, 可以更加以独特的视角进行科技工作, 找出能够更加符合人类社会发展的科技技术; 在思想政治 领域内, 具有良好的审美水平, 就可以站在一个符合社会 社会主流意识形态发展的视角之上, 促使社会进步与发展。

而究其原因来看, 大学生审美素质水平的高低之所以 与创新能力有关, 是基于两者之间的想象力问题。须知审 美素质中先天审美因素和后天审美因素, 先天的审美不消 多说, 因为创新能力与先天有着关联但更多的是与后天因 素有关。而再看后天审美, 前文所述的审美态度、审美情 趣、审美经验, 正确的审美态度是对一个客观主体不含功 利色彩的心态的表征, 而审美情趣则是具有严重的主观色 彩, 又好坏之分, 审美经验则是对经验的积累。而审美与 之想象力的关系看, 想象力是一种人们对事物的一种形象 思维能力, 而审美同样是不同主体在审美活动中的思维方 式, 不同的审美对待同一事物的审美可能完全不一样, 这 就是说不同个体对待同一事物的思维分析进行了二次创 造, 以此看来, 审美的思维兼具了想象力与创造力。而反 观创新的思维, 所必须包好的几个要素, 首先就是具有一 定的想象能力, 然后才能进行在思维上对待事物进行二次 思维创造。两者这样的结合看来, 审美素质的高低可以促 使人的想象力和思维二次创造能力增强, 那么对创新能力 的提高也是有着积极的作用。

当今时代之下, 随着网络文化的盛行甚至于是泛滥, 大学生的思想受到了深深的冲击, 在审美态度之上逐渐变 得功力主义, 这已经严重的偏离了审美素质要求本身; 在 审美情趣上部分变得消极而偏激, 这也同样与审美素质要 求大相径庭; 审美基本要求之上尚不能做到这一点, 更无 需谈审美经验的积累, 审美水平的高低了。在当今中国大 学生在审美水平低下上表现最为明显的特征就是贵乏创新 能力, 这也是许多人对当代大学生的一种社会评价。 


\section{2 大学生的审美素质与其素质全面发展有着深刻的关系}

以审美素质教育的视角上来看, 它是一种对大学生全 面的教育, 它与德智体相互之间关联渗透, 并且相互之间 起着促进作用。尽管从教育的角度看来, 与之其他方面的 素质教育审美教育属于侧重于感情方面的教育, 很难以卷 面的成绩来衡量, 其教育效果看来也难以短期内看出, 但 是从人类发展的思维角度来看, 他却是长期逐步发展的。 它能够滋润人的心灵让人们以更加积极向上、愉悦的心情 去面对未来的困难与挑战, 能够与之个人素质的每一个方 面都起到积极促进作用，具体体现在以下几个方面：

第一，大学生的审美素质的高低与之品德、情操有着 内在的联系。

纵览人性的发展过程中, 人的美德是相辅相成, 美虽 然不能够与之真善划等号, 不能代表美德, 但是在本质上 美却与真善有着统一的特性, 二者在人的品质、行为规范 以及伦理结构上都有着和谐的统一。从我国悠久的历史文 化上便可看出这一点, 荀子曾强调“乐也者可以善民心, 其 感人深, 其移风易俗”, 该提法的意思不言而喻美可以陶治 人心, 可以改变人们外在的不良行为。总言之, 人在的外 在表现, 即品德——给予人们评价的素质, 需要一颗美的 内心, 才能够在情感上给予理性的表达, 体现出来良好的 品德与情操。由一般人性的演绎, 对于大学生而言他们同 样如此其良好的品德情操依然需要一颗美的心。

第二, 大学生的审美素质高低与之智力发展有着内在 的联系。

从认知心理学的视角上看, 智力发育是一种认知上的 过程, 在整个大学生认知发展过程中他们需要在短短大学
几年中既要学习、存储海量的多年人类知识精华。而在这 个学生枯燥的学习过程中, 若有一双善于发现美的双眼, 那么就可以在学生这个认知过程起到调节剂的作用。同时 它还能够丰富整个学习过程中的内容, 将海量的理性知识 以更为感性的知识予以填补, 能够促使在精神变得枯燥的 时候感觉到一丝新鲜的空气, 须知感性思维与理想思维的 最大的区别之处就是在用脑机制之上，这种审美素质教育 能够弥补用脑上的不足，从这个角度上改善了智力发展的 中全面性。这种角度看来, 大学生的审美素质高低与之智 力发展也就有了这方面的内在联系。

纵观当今我国各大高校的大学生, 总体上来说, 他们 的素质都还不错, 但在创新水平上略显不足, 要从教育上 入手解决大学生群体中这个问题, 应以一个柔性的角度去 进行, 而审美素质教育就是这样的一种方法, 能够在不知 不觉中逐渐改善大学生在素质上所欠缺之处。

\section{参考文献(References)}

[1] Qian study of life in any aesthetic conducted among college students learn Weekly: B, 2012, (9) :65-66.

[2] Zhong Shilun, Kang Sheng, Turre, etc. as a starting point to build aesthetic quality of the implementation of the education system. Hebei University (Philosophy and Social Sciences), 2012, 37 (1):5-9.

[3] leaves the sea. University campus culture and aesthetic characteristics of the student population. Heilongjiang Higher Education Research, 2011, (6) :50-53

[4] Xiao Rong, Wu Ziqiang, Wang Yuan, etc. Students' Moral aesthetic value of the activation and implementation path implication. Jiangsu Higher Education, 2011, (4):116-118. 